\title{
Prognostic value of neoadjuvant treatment response in locally advanced esophageal adenocarcinoma
}

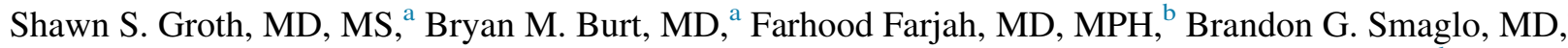
Yvonne H. Sada, MD, MPH, ${ }^{\mathrm{c}}$ David J. Sugarbaker, MD, ${ }^{\mathrm{a}}$ and Nader N. Massarweh, MD, MPH ${ }^{\mathrm{d}, \mathrm{e}}$

\section{ABSTRACT}

Objective: To determine the association between neoadjuvant chemotherapy and chemoradiation therapy on completeness of pathologic response and to assess the impact of primary tumor versus nodal response on survival after esophagectomy.

Methods: Patients aged 18 to 80 years in the National Cancer Data Base (2006-2016) with clinically staged, locally advanced (cT2-4 or cN+) esophageal adenocarcinoma who underwent an R0 esophagectomy after neoadjuvant chemotherapy or chemoradiation therapy were included. Multivariable Cox proportional hazards regression models were constructed to assess the association between treatment response and survival.

Results: Among 2870 patients, there was a significant dose-response association between completeness of response and overall survival: no response (reference), partial response (hazard ratio $[\mathrm{HR}], 0.81 ; 95 \%$ confidence interval [CI], 0.72-0.91), and complete response (HR, 0.55; 95\% CI, 0.47-0.65). Compared with neoadjuvant chemotherapy alone, neoadjuvant chemoradiation was associated with higher pathologic primary tumor $(33.9 \%$ vs $21.3 \% ; P<.001)$ and pathologic nodal response rates $(55.9 \%$ vs $32.7 \% ; P<.001)$. Both a primary and nodal response were associated with improved survival. However, among patients with a primary but no nodal response, primary tumor response was not associated with risk of death (HR, 0.88; 95\% CI, 0.69-1.11). In contrast, among patients who had a nodal but no primary response, the survival benefit of a nodal response was maintained (HR, 0.66; 95\% CI, 0.58-0.76).

Conclusions: Pathologic nodal (rather than primary tumor) response to neoadjuvant therapy is associated with improved survival. These data suggest a need to optimize neoadjuvant strategies associated with more complete nodal response rates and to consider more aggressive adjuvant treatment for patients with residual nodal disease after esophagectomy. (J Thorac Cardiovasc Surg 2019;157:1682-1693)

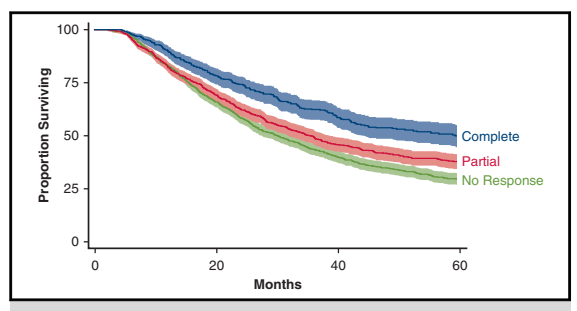

Nodal response to neoadjuvant therapy is independently associated with survival.

\section{Central Message}

Pathologic nodal (rather than primary tumor) response to neoadjuvant therapy for esophageal adenocarcinoma is an independent determinant of survival after esophagectomy with negative margins.

\section{Perspective}

Pathologic nodal (rather than primary tumor) response to neoadjuvant therapy for locally advanced esophageal adenocarcinoma is the principal determinant of survival after a margin-negative esophagectomy. Thus, neoadjuvant therapy should be optimized to maximize nodal response rates, and adjuvant systemic therapy should be considered for patients with residual nodal disease.

See Commentary on page 1694
From the a Baylor College of Medicine, Department of Surgery, Division of General Thoracic Surgery, Houston, Tex; ${ }^{b}$ University of Washington School of Medicine, Department of Surgery, Division of Cardiothoracic Surgery, Seattle, Wash; ${ }^{\mathrm{c} B}$ Baylor College of Medicine, Department of Medicine, Section of Hematology-Oncology, Houston, Tex; ${ }^{\mathrm{d}}$ Center for Innovations in Quality, Effectiveness and Safety, Michael E. DeBakey VA Medical Center, Houston, Tex; and ${ }^{\mathrm{e}}$ Baylor College of Medicine, Department of Surgery, Division of Surgical Oncology, Houston, Tex.

Read at the 98th Annual Meeting of The American Association for Thoracic Surgery, San Diego, California, April 28-May 1, 2018.

Received for publication April 2, 2018; revisions received Nov 9, 2018; accepted for publication Nov 14, 2018; available ahead of print Jan 31, 2019

Address for reprints: Shawn S. Groth, MD, MS, Division of Thoracic Surgery, Michael E. DeBakey Department of Surgery, One Baylor Plaza, BCM 390, Houston, TX 77030 (E-mail: Shawn.Groth@bcm.edu).

$0022-5223 / \$ 36.00$

Copyright (c) 2018 by The American Association for Thoracic Surgery

https://doi.org/10.1016/j.jtcvs.2018.11.131
There have been numerous contemporaneous improvements to the management of esophageal cancer, including endoscopic resection of early-stage tumors, the introduction of biomarker-directed systemic therapies (ie, Her-2, mismatch repair deficiency, microsatellite instability-high, and programmed death-ligand 1), increasing sophistication

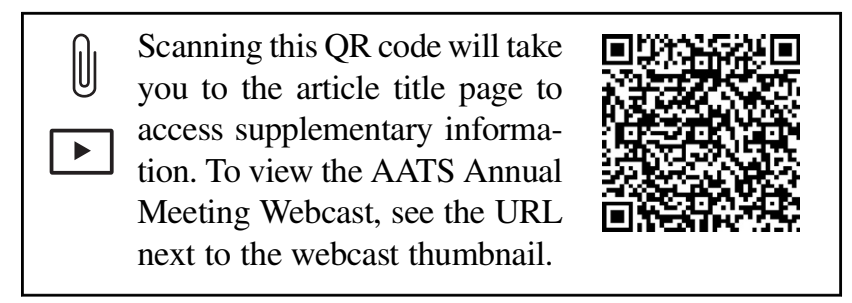




$$
\begin{aligned}
& \text { Abbreviations and Acronyms } \\
& \begin{aligned}
\mathrm{CI} & =\text { confidence interval } \\
\mathrm{CoC} & =\text { Commission on Cancer } \\
\mathrm{HR} & =\text { hazard ratio } \\
\mathrm{NCDB} & =\text { National Cancer Data Base } \\
\mathrm{OS} & =\text { overall survival } \\
\mathrm{RRR} & =\text { relative risk ratio }
\end{aligned}
\end{aligned}
$$

of radiation techniques, and surgical and technologic advances such as minimally invasive approaches to radical resection. Despite these and other refinements to cancer-directed therapy, survival for patients with esophageal cancer has improved little over the past several decades. Even with aggressive multimodal therapy incorporating chemotherapy, radiation therapy, and esophagectomy, the 5-year survival remains $30 \%$ to $50 \%{ }^{1-3}$ Two important factors have been identified that positively affect the outcome of patients diagnosed with esophageal cancer: (1) use of multimodal therapy and (2) disease response to neoadjuvant therapy.

Compared with surgery alone, the addition of neoadjuvant therapy prior to esophagectomy is associated with higher margin negative resection rates, lower recurrence rates, and improved survival. ${ }^{1-3}$ In addition, the degree of pathologic response to neoadjuvant therapy has significant prognostic implications-a complete pathologic response to neoadjuvant therapy is independently associated with improved survival. ${ }^{4-9}$ However, these data regarding the association between pathologic response to neoadjuvant treatment and survival are primarily limited to singleinstitution studies. ${ }^{10}$ Furthermore, prior studies evaluated treatment response as a binary outcome (complete vs incomplete response) with little information available about the impact of partial, as compared with no or complete pathologic response. Furthermore, given a paucity of data from the general community, the generalizability of the prognostic value of pathologic response remains unclear.

The objectives of this study were to evaluate the association between the degree of pathologic response and the overall survival (OS) and to characterize the relative impact of primary tumor response and nodal response on survival after esophagectomy following neoadjuvant therapy. We hypothesize that the degree of pathologic response (eg, no, partial, or complete) to neoadjuvant treatment is associated with survival and that pathologic nodal response to neoadjuvant therapy has a more significant impact on survival than a primary tumor response.

\section{MATERIALS AND METHODS Study Subjects}

This was a retrospective cohort study using the National Cancer Data Base (NCDB). The NCDB is a jointly sponsored project of the Commission on Cancer $(\mathrm{CoC})$ of the American College of Surgeons and the American
Cancer Society. It is a prospectively maintained hospital-based cancer registry containing information on more than $70 \%$ of incident malignancies diagnosed in the United States annually at more than $1500 \mathrm{CoC}-$ accredited centers.

We selected patients aged 18 to 80 years from the NCDB for inclusion in our study using International Classification Disease for Oncology, 3rd Edition codes for esophageal adenocarcinoma. Patients diagnosed with locally advanced (cT2-4 or $\mathrm{cN}+$ ) esophageal adenocarcinoma between 2006 and 2012 and who underwent neoadjuvant chemotherapy or chemoradiation therapy followed by esophagectomy were included.

Exclusion criteria for our cohort are shown in Figure 1. Patients with esophageal squamous cell carcinoma were excluded because it accounts for the minority of esophageal cancer diagnoses in the United States and because of its notable differences in response rate to neoadjuvant therapy compared with adenocarcinoma. ${ }^{1}$ We excluded patients who had a positive or unknown resection margin $(\mathrm{n}=393)$. We also excluded patients who were treated outside of the reporting facility to improve the accuracy of the ascertainment of the receipt all neoadjuvant and adjuvant therapies.

This study was approved by the Human Subjects Committee of the Baylor College of Medicine Institutional Review Board and the Michael E. DeBakey VA Medical Center Research and Development Committee.

\section{Definitions}

Disease response to neoadjuvant therapy was the main variable of interest in this study. Response rates were classified on the basis of the degree of response: 1. complete response (ypT0N0); 2. partial response-primary tumor or nodal downstaging (specifically, cT3 to ypTis-1 or cT4 to ypTis-2 regardless of pathologic nodal status, $\mathrm{cN}+$ to ypNO with residual primary tumor, or ypN + with a complete primary response); 3. no response. This definition for primary tumor response was chosen because it should conservatively bias results toward the null. Patients were also categorized on the basis of the type of neoadjuvant treatment received. The NCDB does not provide information to differentiate between patients treated with neoadjuvant concurrent chemoradiation therapy and neoadjuvant sequential chemotherapy and (chemo)radiation therapy. All patients in our cohort were treated with neoadjuvant chemotherapy. Consequently, patients were classified as having been treated with neoadjuvant radiation or not.

\section{Statistical Analysis}

Continuous and categoric variable distributions were evaluated with standard descriptive statistics. The primary outcome was OS, and distributions across disease response categories were evaluated using the Kaplan-Meier method and log-rank test. The association between the use of neoadjuvant radiation and the disease response was evaluated using multivariable, multinomial regression. Multivariable Poisson regression was used to evaluate the association between the use of neoadjuvant radiation and the primary tumor and nodal responses separately ${ }^{11-14}$ The association between risk of death and pathologic disease response was examined with multivariable Cox regression. The proportional hazards assumption was evaluated graphically. Because $90.7 \%$ of patients in the study cohort received neoadjuvant radiation and NCDB does not provide information pertaining to the reasons a patient was treated with chemotherapy alone, Cox models were limited to patients who received neoadjuvant radiation. To evaluate the relative impact of primary tumor response and nodal response on risk of death, we separately evaluated patients who had a primary tumor response (with and without nodal response) and patients who had a nodal response (with and without primary tumor response). In all models, covariates were empirically selected nonparsimoniously and included age, sex, race, insurance status, income, education, rurality, Charlson comorbidity score, type of treating facility, tumor grade, and receipt of adjuvant therapy. All models included an adjustment for clustering of patients within hospitals through the use of robust standard errors. 


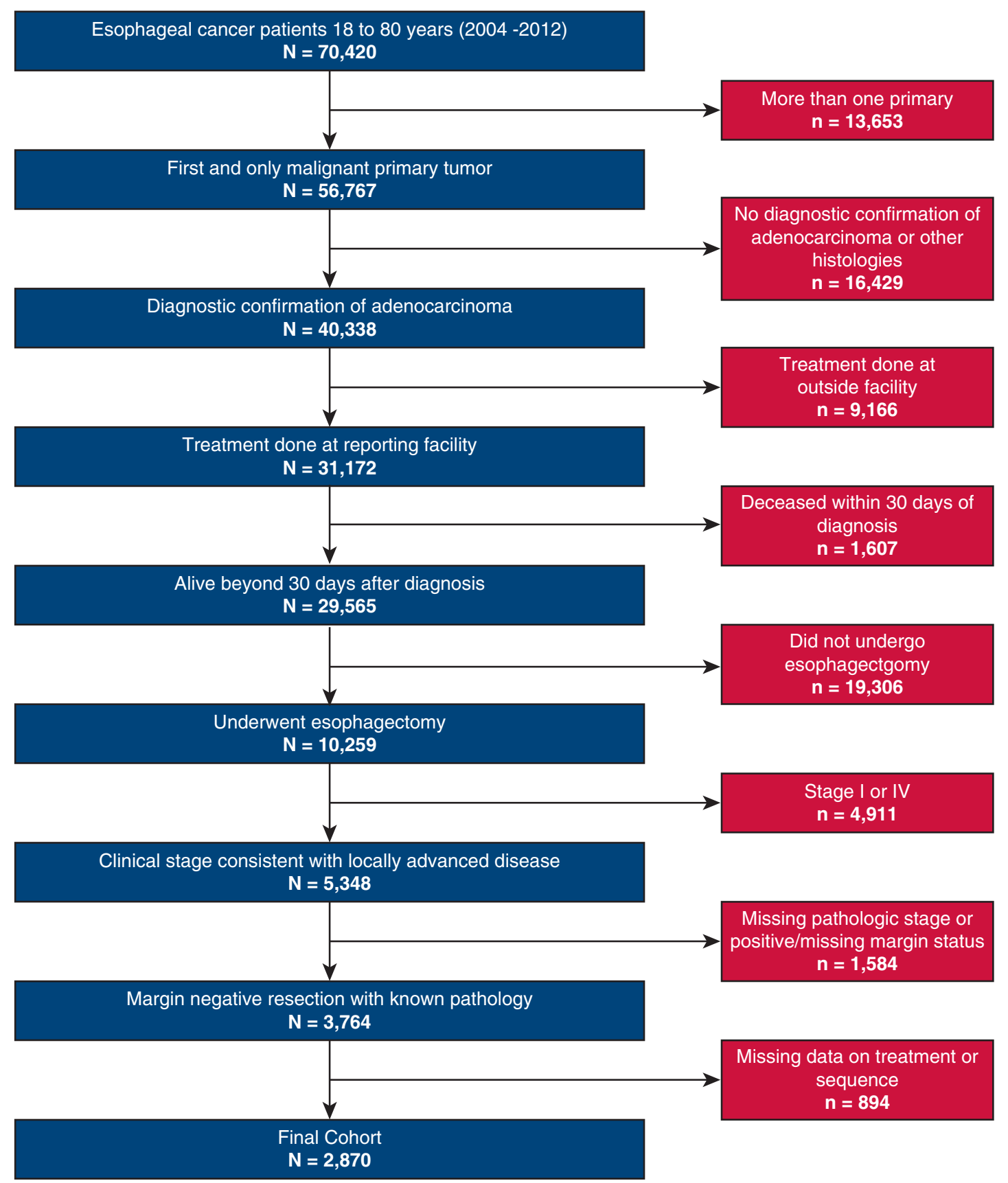

FIGURE 1. Flow diagram illustrating the inclusion and exclusion criteria that were used to arrive at the final study cohort from all patients with esophageal cancer in the NCDB (2004-2012).

Four additional sensitivity analyses were performed. First, because prior work has demonstrated a benefit for the use of adjuvant therapy in select patients, we restricted our Cox models to those who did not receive any adjuvant treatment $(\mathrm{n}=2569) .{ }^{15}$ Second, to address the potential impact of the quality of surgery and the accuracy of pathologic nodal staging, we also restricted our models to patients who had 15 or more lymph nodes pathologically evaluated $(\mathrm{n}=1092) .{ }^{16}$ Third, to ensure that our findings were not explained by early deaths among patients with no response or partial response, we applied a 90-day landmark analysis to the overall Cox model. ${ }^{17}$ Finally, to address possible secular trends in care that may have been associated with improved outcomes, year of diagnosis was included as a model covariate.

Missing covariate data were present for $19.6 \%$ of the cohort. However, 5 -year OS was not significantly different between those who did and did not have missing data in the overall cohort (log-rank, $P=.31$ ) or among those with varying degrees of disease response (no response-log-rank, $P=.41$; partial response-log-rank, $P=.40$; complete response- $\log$-rank, $P=.37$ ). To address missing values, models were run using a case-complete approach and after multiple imputation by chained equations. Results of both approaches were similar, and results from the 
TABLE 1. Demographic and clinical characteristics of the study cohort stratified by type of pathologic response to neoadjuvant treatment

\begin{tabular}{|c|c|c|c|c|}
\hline & No response $(n=1383)$ & Incomplete response $(\mathrm{n}=991)$ & Complete response $(n=496)$ & $P$ value \\
\hline \multicolumn{5}{|l|}{ Demographic } \\
\hline Mean age $\pm S D, y$ & $60.3 \pm 9.3$ & $61.0 \pm 9.2$ & $61.3 \pm 9.1$ & .02 \\
\hline $\begin{array}{l}\text { Age, y }(\%) \\
\quad \leq 55 \\
56-60 \\
61-65 \\
>65\end{array}$ & $\begin{array}{l}30.1 \\
17.9 \\
21.6 \\
30.4\end{array}$ & $\begin{array}{l}26.9 \\
17.6 \\
21.8 \\
33.7\end{array}$ & $\begin{array}{l}24.2 \\
15.7 \\
23.4 \\
36.7\end{array}$ & .07 \\
\hline Male sex $(\%)$ & 89.6 & 90.3 & 90.3 & .81 \\
\hline $\begin{array}{l}\text { Race }(\%) \\
\text { White } \\
\text { Nonwhite } \\
\text { Missing }\end{array}$ & $\begin{array}{r}95.8 \\
2.6 \\
1.6\end{array}$ & $\begin{array}{r}96.3 \\
2.9 \\
0.8\end{array}$ & $\begin{array}{r}97.0 \\
2.4 \\
0.6\end{array}$ & .28 \\
\hline $\begin{array}{l}\text { Insurance status (\%) } \\
\text { Insured } \\
\text { Medicare/Medicaid } \\
\text { Other } \\
\text { Missing }\end{array}$ & $\begin{array}{r}55.8 \\
39.3 \\
3.8 \\
1.2\end{array}$ & $\begin{array}{r}55.6 \\
40.0 \\
4.0 \\
0.4\end{array}$ & $\begin{array}{r}56.1 \\
40.9 \\
2.8 \\
0.2\end{array}$ & .16 \\
\hline $\begin{array}{l}\text { Income }(\%)^{*} \\
\geq \$ 63,000 \\
\text { Missing }\end{array}$ & $\begin{array}{r}32.8 \\
1.6\end{array}$ & $\begin{array}{r}34.6 \\
1.9\end{array}$ & $\begin{array}{r}32.1 \\
1.2\end{array}$ & .63 \\
\hline $\begin{array}{l}\text { Education }(\%)^{*} \\
\quad<7 \% \\
\text { Missing }\end{array}$ & $\begin{array}{r}26.0 \\
1.6\end{array}$ & $\begin{array}{r}28.4 \\
1.8\end{array}$ & $\begin{array}{r}32.5 \\
1.2\end{array}$ & .08 \\
\hline $\begin{array}{l}\text { Rurality (\%) } \\
\text { Metropolitan } \\
\text { Urban } \\
\text { Rural } \\
\text { Missing }\end{array}$ & $\begin{array}{r}45.0 \\
31.2 \\
20.6 \\
3.2\end{array}$ & $\begin{array}{r}47.8 \\
32.1 \\
16.5 \\
3.6\end{array}$ & $\begin{array}{r}44.8 \\
34.3 \\
17.7 \\
3.2\end{array}$ & .22 \\
\hline $\begin{array}{l}\text { Comorbidity index (\%) } \\
\quad 0 \\
1 \\
\geq 2\end{array}$ & $\begin{array}{r}74.8 \\
20.6 \\
4.6\end{array}$ & $\begin{array}{r}73.7 \\
21.4 \\
4.9\end{array}$ & $\begin{array}{r}78.0 \\
19.2 \\
2.8\end{array}$ & .26 \\
\hline $\begin{array}{l}\text { Facility } \\
\text { Hospital type }(\%) \\
\text { Academic/research } \\
\text { Comprehensive cancer center } \\
\text { Other } \\
\text { Missing }\end{array}$ & $\begin{array}{r}58.1 \\
28.7 \\
11.3 \\
2.0\end{array}$ & $\begin{array}{r}62.4 \\
27.7 \\
8.3 \\
1.7\end{array}$ & $\begin{array}{r}65.7 \\
26.8 \\
5.7 \\
1.8\end{array}$ & .004 \\
\hline \multicolumn{5}{|l|}{ Clinical } \\
\hline $\begin{array}{l}\text { Grade }(\%) \\
\text { Well/moderate } \\
\text { Poor/undifferentiated } \\
\text { Missing }\end{array}$ & $\begin{array}{l}37.6 \\
51.7 \\
10.7\end{array}$ & $\begin{array}{l}40.2 \\
46.6 \\
13.2\end{array}$ & $\begin{array}{l}34.9 \\
43.6 \\
21.6\end{array}$ & $<.001$ \\
\hline $\begin{array}{l}\text { Clinical T stage (\%) } \\
\begin{array}{l}1 \\
2 \\
3 \\
4\end{array}\end{array}$ & $\begin{array}{r}2.3 \\
22.1 \\
73.3 \\
2.4\end{array}$ & $\begin{array}{r}2.8 \\
15.4 \\
77.1 \\
4.6\end{array}$ & $\begin{array}{r}2.8 \\
26.0 \\
67.5 \\
3.6\end{array}$ & $<.001$ \\
\hline $\begin{array}{l}\text { Clinical N stage }(\%) \\
\text { Positive }\end{array}$ & 54.0 & 86.5 & 66.9 & $<.001$ \\
\hline
\end{tabular}


TABLE 1. Continued

\begin{tabular}{|c|c|c|c|c|}
\hline & No response $(n=1383)$ & Incomplete response $(n=991)$ & Complete response $(n=496)$ & $P$ value \\
\hline Pathologic T stage (\%) & & & & $<.001$ \\
\hline 0 & 0 & 9.0 & 100 & \\
\hline In situ & 0 & 2.8 & 0 & \\
\hline 1 & 7.6 & 38.2 & 0 & \\
\hline 2 & 26.6 & 22.3 & 0 & \\
\hline 3 & 64.6 & 27.3 & 0 & \\
\hline 4 & 1.2 & 0.4 & 0 & \\
\hline Pathologic $\mathrm{N}+(\%)$ & & & & $<.001$ \\
\hline Positive & 69.1 & 17.6 & 0 & \\
\hline Neoadjuvant treatment $(\%)$ & & & & $<.001$ \\
\hline Radiation therapy used & 87.3 & 92.6 & 96.2 & \\
\hline Chemotherapy only & 12.7 & 7.4 & 3.8 & \\
\hline Chemotherapy regimen $(\%)$ & & & & .24 \\
\hline Single-agent & 5.2 & 3.9 & 4.4 & \\
\hline Multi-agent & 83.5 & 86.9 & 83.1 & \\
\hline Chemotherapy administered, NOS & 11.2 & 9.2 & 12.5 & \\
\hline Adjuvant treatment $(\%)$ & & & & $<.001$ \\
\hline Radiation therapy used & 1.5 & 0.1 & 0 & \\
\hline Chemotherapy only & 12.9 & 8.2 & 4.0 & \\
\hline
\end{tabular}

SD, Standard deviation; NOS, not otherwise specified; . *Based on 2008-2012 American Community Survey data. For income, the percentage of patients whose area of residence (based on ZIP code) had a median household income $\$ 63,000$ or greater (adjusted for 2012 inflation) is presented. For education, the percentage of patients whose area of residence (based on ZIP code) had less than 7\% adults who did not attain a high school education is presented.

imputed models are presented. Analyses were performed using STATA version 14.0 (StataCorp, LP, College Station, Tex).

\section{RESULTS}

\section{Study Cohort}

The characteristics of the 2870 patients included in the cohort are presented in Table 1. Overall, $17.3 \%$ had a complete response, $34.5 \%$ had a partial response, and $48.2 \%$ had no response. The majority of patients in all disease response categories received a multi-agent chemotherapy regimen. Among those treated with neoadjuvant chemotherapy alone, $79.5 \%$ received a multiagent regimen. Adjuvant therapy was used less frequently as the degree of pathologic response to neoadjuvant therapy increased (no response, $14.4 \%$; partial response, $8.3 \%$; complete response, $4.0 \%$ ). There was no significant difference in the 30-day (neoadjuvant radiation, 2.9\%; neoadjuvant chemotherapy, $2.2 \% ; P=.52$ ) or 90 -day (neoadjuvant radiation, $7.0 \%$; neoadjuvant chemotherapy, $5.6 \% ; P=.38$ ) mortality between neoadjuvant treatment types.

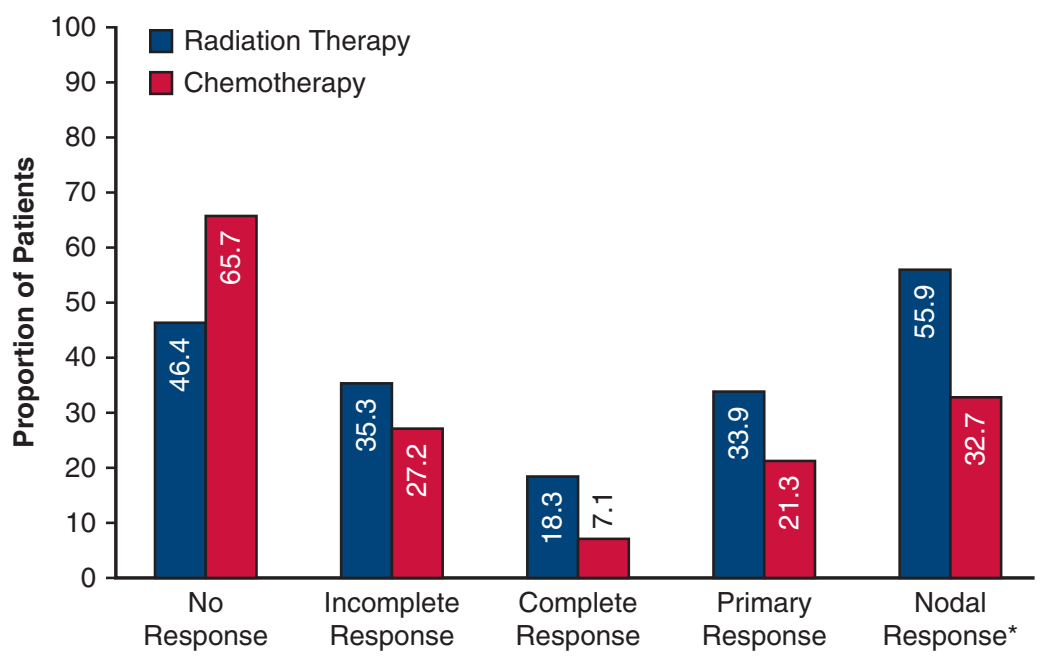

FIGURE 2. Disease response rates to neoadjuvant chemotherapy alone (chemotherapy) versus neoadjuvant chemoradiation therapy (radiation therapy), defined as complete response (ypT0N0), partial response (primary tumor or nodal downstaging, specifically, cT3 to ypTis-1 or cT4 to ypTis-2 regardless of pathologic nodal status, $\mathrm{cN}+$ to ypN0 with residual primary tumor, or ypN + with a complete primary response), and no response. Primary response rates were ascertained among node-negative patients. Nodal response rates were ascertained among clinically node-positive patients. 


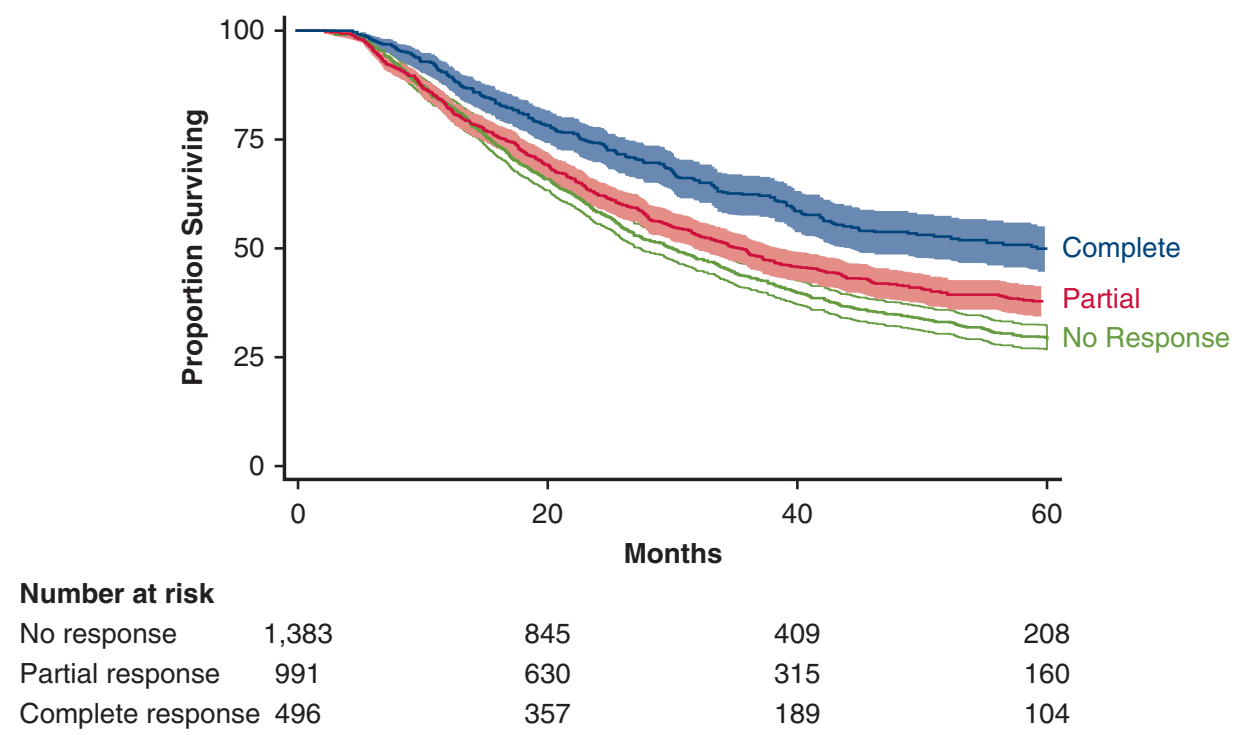

FIGURE 3. OS (and 95\% CIs) based on the degree of pathologic response to neoadjuvant therapy, defined as complete response (ypT0N0), partial response (primary tumor or nodal downstaging, specifically, cT3 to ypTis-1 or cT4 to ypTis-2 regardless of pathologic nodal status, $\mathrm{cN}+$ to ypN0 with residual primary tumor, or $\mathrm{ypN}+$ with a complete primary response), and no response.

\section{Association Between Neoadjuvant Therapy and Response to Therapy}

Higher rates of complete $(18.3 \%$ vs $7.1 \%, P<.001)$ and partial response $(35.3 \%$ vs $27.2 \%, P=.008)$ were observed with neoadjuvant radiation compared with chemotherapy alone (Figure 2). In addition, primary tumor response rates were higher in patients who received neoadjuvant radiation compared with neoadjuvant chemotherapy alone $(33.9 \%$ vs $21.3 \%, P<.001)$. Furthermore, among patients with clinically node positive disease, nodal downstaging was more common after neoadjuvant radiation compared with chemotherapy alone $(55.9 \%$ vs $32.7 \%, P<.001)$. Compared with patients who received neoadjuvant radiation, patients who received neoadjuvant chemotherapy alone were less likely to have a partial (relative risk ratio [RRR], $0.52 ; 95 \%$ confidence interval [CI], 0.40-0.68) or complete response (RRR, 0.26; 95\% CI, 0.16-0.42). Compared with patients who received neoadjuvant radiation, patients who received neoadjuvant chemotherapy alone were less likely to have a complete primary tumor response (RRR, $0.63 ; 95 \%$ CI, 0.48-0.81) or a complete nodal response (RRR, $0.56 ; 95 \% \mathrm{CI}$, 0.46-0.69).

\section{Association Between Response to Therapy and Survival}

For patients who were treated with neoadjuvant radiation therapy, the 5-year OS increased with the degree of treatment response (no response 29.3\%; partial response $37.8 \%$; complete response $49.9 \% ; P<.001$ ) (Figure 3). Even after adjusting for potential confounding variables, complete (hazard ratio [HR], 0.54; 95\% CI, 0.46-0.63) and partial (HR, 0.80; 95\% CI, 0.71-0.91) pathologic responses were associated with a significantly decreased overall risk of death compared with patients who had no response (Table 2). These findings were similar when limiting the analysis to patients who did not receive any adjuvant therapy, those who had an adequate lymphadenectomy, in the landmark analysis, and when adjusting for year of diagnosis (data not shown).

\section{Relative Contribution of Primary Tumor Response Versus Nodal Response on Overall Survival}

Patients with a primary tumor response had a better 5year OS than patients without a primary tumor response ( $44.3 \%$ vs $31.6 \%$, log-rank, $P<.001)$. However, among patients with residual nodal disease, primary tumor response was not associated with improved unadjusted 5-year OS $(27.0 \%$ vs $21.9 \%$, log-rank, $P=.19)$. After adjusting for potential confounding variables, the overall risk of death was significantly lower among those who had a complete primary tumor response (HR, 0.71; 95\% CI, 0.63-0.79), but the magnitude of this association was mitigated among those who did not have a pathologically complete nodal response (HR, 0.88; 95\% CI, 0.69-1.12) (Table 2). Compared with patients who had residual nodal disease, clinically node positive patients who achieved a complete nodal response had better 5 -year OS $(42.3 \%$ vs $21.8 \%$; log-rank, $P<.001$ ), even among those patients who did not have a complete primary tumor response $(37.5 \%$ vs $21.0 \%$; log-rank, $P<.001)$. After adjusting for potential confounding variables, the risk of death was also significant lower in those who had a complete nodal response (HR, 0.60; 95\% CI, 0.54-0.67), regardless of whether there 
TABLE 2. Association between degree and type of disease response and overall risk of death among patients who underwent a margin-negative esophagectomy after neoadjuvant chemotherapy and radiation for locally advanced esophageal adenocarcinoma*

\begin{tabular}{|cc}
\hline & Hazard ratio $(\mathbf{9 5} \% \mathbf{C I})$ \\
\hline Overall cohort & Reference \\
No response & $0.80(0.71-0.91)$ \\
Partial response & $0.54(0.46-0.63)$ \\
Complete response & Reference \\
No adjuvant therapy & $0.81(0.71-0.93)$ \\
$\quad$ No response & $0.54(0.46-0.63)$ \\
Partial response & \\
Complete response & Reference \\
$\geq 15$ lymph nodes examined & $0.72(0.58-0.91)$ \\
$\quad$ No response & $0.55(0.42-0.73)$ \\
Partial response & \\
Complete response & Reference \\
Primary tumor response & $0.70(0.62-0.79)$ \\
Incomplete primary response & \\
Complete primary response & Reference \\
\hline Primary tumor response and no nodal response & \\
Incomplete primary response & $0.88(0.70-1.12)$ \\
Complete primary response & \\
\hline Nodal response & Reference \\
No nodal response & $0.58(0.52-0.65)$ \\
Nodal response & \\
\hline Node response and no primary response & $0.64(0.55-0.74)$ \\
\hline No nodal response & \\
Nodal response & \\
\hline
\end{tabular}

CI, Confidence interval. *All models were adjusted for age, sex, race, insurance status, income, education, rurality, Charlson comorbidity score, type of treating facility, tumor grade, and receipt of adjuvant therapy. The full model for the overall cohort is provided in Table E1.

was a complete primary response (HR, 0.66; 95\% CI, 0.57-0.76) (Table 2).

\section{DISCUSSION}

Compared with surgery alone, there is a clear benefit associated with esophagectomy as part of a multimodal treatment strategy (along with chemotherapy with or without radiation therapy) for patients with locally advanced esophageal adenocarcinoma. ${ }^{1-3,18-20}$ Largely based on the results of the CROSS trial, concurrent neoadjuvant chemoradiation therapy followed by esophagectomy has become the preferred treatment strategy of most centers in the United States for patients with locally advanced disease. The findings of our national cohort of patients with esophageal adenocarcinoma support several important observations with regard to patients who undergo neoadjuvant therapy followed by an esophagectomy with negative margins.

First, compared with neoadjuvant chemotherapy alone, the combination of neoadjuvant chemotherapy and radiation therapy is associated with higher pathologic response rates. Samson and colleagues ${ }^{9}$ also used data from the NCBD and found that the use of neoadjuvant radiation was associated with higher pathologic complete response rates $(17.2 \%)$ compared with neoadjuvant chemotherapy alone $(6.4 \%)$. In contrast to the study by Samson and colleagues ${ }^{9}$ and other single institution studies $^{4-8}$ that have examined pathologic response as a binary outcome (complete response vs no/partial response), we noted a clear dose-response relationship between the degree of pathologic response (no, partial, and complete response) and OS. This dose-response observation has potentially important implications for patient care by providing more detailed prognostic information for counseling patients, personalizing the amount of neoadjuvant chemotherapy, selecting patients who may benefit from adjuvant therapy, and guiding post-treatment surveillance.

Second, independent of primary tumor response, nodal response to neoadjuvant chemotherapy and radiation therapy appears to be the principle determinant of survival after an R0 esophagectomy. These findings are consistent with recently published follow-up data from the MAGIC trial, which compared perioperative chemotherapy and surgery versus surgery alone for resectable gastroesophageal adenocarcinomas, $24 \%$ of which were distal esophageal or gastroesophageal junction tumors. The authors of this trial found that the presence of lymph node metastasis was the only independent predictor of OS (HR, 3.36; 95\% CI, 1.70-6.63). ${ }^{21}$ Data from other observational studies ${ }^{10,22-25}$ and clinical trials ${ }^{18}$ confirm the association between lymphovascular invasion or residual nodal disease and poorer survival. However, compared with data from clinical trials or quaternary referral centers, our data are derived from the general population, which enhances the generalizability of these findings.

Higher recurrence rates likely underlie the association between residual nodal disease and poorer survival. ${ }^{22,23}$ Indeed, results from a phase II clinical trial comparing perioperative chemotherapy and surgery with surgery alone for distal esophageal or gastroesophageal junction carcinomas demonstrated recurrence was more common in node-positive $(55 \%)$ than in node-negative $(22 \%)$ patients. ${ }^{26}$ In particular, a multivariate analysis of CROSS trial data demonstrated that pathologic nodal status, not primary tumor stage, was independently associated with an approximately 3-fold increased risk of locoregional recurrence. ${ }^{19}$ Unfortunately, the NCDB does not provide recurrence data to researchers for us to further assess the impact of nodal disease on recurrence rates.

In the context of these data, our results could have several important implications for clinical practice. At present, the role of adjuvant chemotherapy for patients who undergo an R0 esophagectomy after neoadjuvant therapy is unclear. ${ }^{23}$ However, our data are consistent with previous work 
suggesting patients with residual nodal disease are at a higher risk of recurrence and lower survival and suggest that these patients may benefit from more aggressive systemic therapy. ${ }^{22,27}$ Indeed, although there are presently no published randomized data regarding the selection of patients for adjuvant therapy, our group previously demonstrated a survival benefit associated with adjuvant chemotherapy limited to patients who have residual nodal disease after neoadjuvant chemoradiation therapy. ${ }^{15}$

Although patients with residual nodal disease may theoretically benefit from adjuvant chemotherapy, many such patients never receive it. Among patients in the MAGIC trial assigned to the perioperative chemotherapy arm, only $42 \%$ completed all assigned treatments, and $34 \%$ of patients who completed neoadjuvant therapy and surgery did not receive adjuvant chemotherapy because of early disease progression, postoperative complications, or patient refusal. ${ }^{2}$ Likewise, in a Federation Nationale des Centres de Lutte Contre Le Cancer/Federation Francophone de Cancerologie multicenter phase III trial, which compared perioperative chemotherapy and surgery versus surgery alone for resectable gastroesophageal adenocarcinomas, only $50 \%$ of patients assigned to perioperative chemotherapy received at least 1 cycle of adjuvant chemotherapy and only $39 \%$ completed all assigned treatments. ${ }^{3}$ Because of similar limitations in administering adjuvant therapy in the treatment of patients with other locally advanced, resectable gastrointestinal malignancies, there is increasing interest in more aggressive "total upfront" neoadjuvant strategies in pancreatic ductal adenocarcinoma,${ }^{28}$ gastric adenocarcinoma, ${ }^{29}$ and rectal adenocarcinoma. ${ }^{30}$ Given the aggressive nature of esophageal cancer, our inability to predict which patients will respond to neoadjuvant therapy, and the morbidity associated with surgical resection that currently limits the number of patients who are able to receive adjuvant treatment, certain patients with locally advanced esophageal adenocarcinoma may also benefit from this "total upfront" approach before esophagectomy. Most cancer-related deaths from esophageal carcinoma are due to systemic disease. For patients who would likely benefit from systemic therapy, history has taught us that most patients $(\sim 60 \%)$ cannot complete a full planned course of adjuvant chemotherapy using currently available regimens. Therefore, consideration should be given to optimizing nodal response rates in the neoadjuvant setting.

Given the existing data regarding the importance of pathologic nodal status, one possible approach could be to use a given patient's nodal status to guide the use of additional therapy in the neoadjuvant setting. For example, if a clinically node-positive patient was treated with neoadjuvant chemoradiation and on restaging found have residual nodal disease, consideration could be given to providing additional systemic therapy in an effort to convert

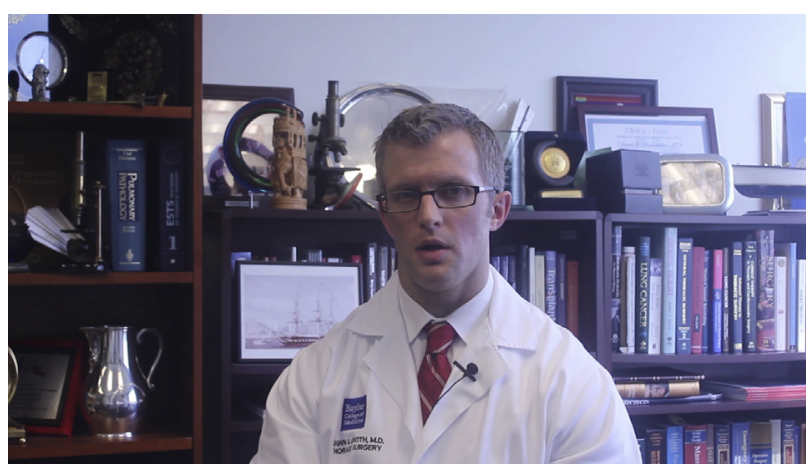

VIDEO 1. Dr Shawn Groth summarizing the findings of this study. Video available at: https://www.jtcvs.org/article/S0022-5223(18)33307-5/ fulltext.

that patient to node-negative disease rather than proceeding immediately to esophagectomy. Likewise, for patients who are initially clinically node negative but demonstrate progressive disease after neoadjuvant therapy, consideration could be given to providing additional systemic treatment (perhaps an alternative regimen or targeted therapy) before proceeding with radical resection. Cancer and Leukemia Group B (Alliance) 80803 is a cooperative group trial that is evaluating the concept of adjusting neoadjuvant chemotherapy before esophagectomy based on the patient's clinical response. ${ }^{31}$ Perhaps patients who are nonresponders would benefit from biomarker-directed neoadjuvant targeted therapy or immunotherapy. A clinical trial to further evaluate such strategies is in order.

\section{Study Limitations}

There are several limitations of our study. The NCDB does not provide information on clinical decision-making; thus, we could not ascertain the reasons patients were treated with neoadjuvant chemotherapy alone or with radiation. Therefore, selection bias is a potential limitation of our study. Although unmeasured differences in performance status (which is not available in NCDB) may have played a role in treatment decisions or eventual outcome, we noted no difference in 30- or 90-day mortality rates between patients treated with either type of neoadjuvant therapy. Furthermore, the consistency of our results across all sensitivity analyses and in our landmark analysis speaks to the robustness of our findings to varying assumptions. To assess treatment response, we compared pretreatment clinical staging with pathologic staging. The limitations of clinical staging could introduce error into our analysis in patients who had a partial response. Therefore, we chose a relatively conservative definition for partial response to conservatively bias our results toward the null. The NCDB does not collect information on several patient factors that are associated with survival, such as margin length, ${ }^{32}$ local and distant recurrence, ${ }^{33}$ and 
performance status before and after treatment. In addition, the NCDB does not collect data on postoperative complications, which may affect cancer-specific survival. ${ }^{34}$ Although detailed data on the specific chemotherapy regimen and number of cycles administered are not provided, the majority of patients in our cohort were treated with multi-agent chemotherapy, which would be consistent with the regimens used in the MAGIC or CROSS trials. Information on the cause of death is not provided; thus, cancer-specific survival could not be calculated. Data regarding the staging modalities used to ascertain clinical stage are not available. Finally, the NCDB only collects data from $\mathrm{CoC}$-accredited hospitals, which could affect the generalizability of our findings. 35,36

\section{CONCLUSIONS}

Pathologic nodal (rather than primary tumor) response to neoadjuvant therapy for locally advanced esophageal adenocarcinoma is the principal determinant of survival after an esophagectomy with negative margins (Video 1).

\section{Webcast}

You can watch a Webcast of this AATS meeting presentation by going to: https://aats.blob.core.windows. net/media/18May01/25ABC\%202.General\%20Thoracic $\%$ 20SS/S91\%20-\%20Part \%202/S91_3_webcast_04360 7106.mp4.

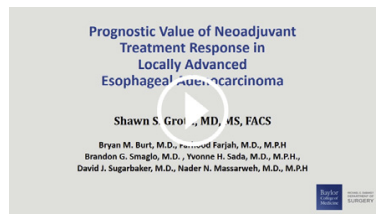

\section{Conflict of Interest Statement}

Authors have nothing to disclose with regard to commercial support.

\section{References}

1. van Hagen P, Hulshof MC, van Lanschot JJ, Steyerberg EW, van Berge Henegouwen MI, Wijnhoven BP, et al. Preoperative chemoradiotherapy for esophageal or junctional cancer. N Engl J Med. 2012;366:2074-84.

2. Cunningham D, Allum WH, Stenning SP, Thompson JN, Van de Velde CJ, Nicolson M, et al. Perioperative chemotherapy versus surgery alone for resectable gastroesophageal cancer. $N$ Engl J Med. 2006;355:11-20.

3. Ychou M, Boige V, Pignon JP, Conroy T, Bouche O, Lebreton G, et al Perioperative chemotherapy compared with surgery alone for resectable gastroesophageal adenocarcinoma: an FNCLCC and FFCD multicenter phase III trial. J Clin Oncol. 2011;29:1715-21.

4. Alnaji RM, Du W, Gabriel E, Singla S, Attwood K, Nava H, et al. Pathologic complete response is an independent predictor of improved survival following neoadjuvant chemoradiation for esophageal adenocarcinoma. J Gastrointest Surg. 2016;20:1541-6.

5. Berger AC, Farma J, Scott WJ, Freedman G, Weiner L, Cheng JD, et al Complete response to neoadjuvant chemoradiotherapy in esophageal carcinoma is associated with significantly improved survival. J Clin Oncol. $2005 ; 23: 4330-7$.
6. Donahue JM, Nichols FC, Li Z, Schomas DA, Allen MS, Cassivi SD, et al. Complete pathologic response after neoadjuvant chemoradiotherapy for esophageal cancer is associated with enhanced survival. Ann Thorac Surg. 2009;87:392-9.

7. Hammoud ZT, Kesler KA, Ferguson MK, Battafarrano RJ, Bhogaraju A, Hanna N, et al. Survival outcomes of resected patients who demonstrate a pathologic complete response after neoadjuvant chemoradiation therapy for locally advanced esophageal cancer. Dis Esophagus. 2006;19:69-72.

8. Meredith KL, Weber JM, Turaga KK, Siegel EM, McLoughlin J, Hoffe S, et al. Pathologic response after neoadjuvant therapy is the major determinant of survival in patients with esophageal cancer. Ann Surg Oncol. 2010;17: 1159-67.

9. Samson P, Robinson C, Bradley J, Lockhart AC, Puri V, Broderick S, et al. Neoadjuvant chemotherapy versus chemoradiation prior to esophagectomy: impact on rate of complete pathologic response and survival in esophageal cancer patients. J Thorac Oncol. 2016;11:2227-37.

10. Gaca JG, Petersen RP, Peterson BL, Harpole DH Jr, D'Amico TA, Pappas TN, et al. Pathologic nodal status predicts disease-free survival after neoadjuvant chemoradiation for gastroesophageal junction carcinoma. Ann Surg Oncol. 2006;13:340-6.

11. Massarweh NN, Park JO, Yeung RS, Flum DR. Comparative assessment of the safety and effectiveness of radiofrequency ablation among elderly Medicare beneficiaries with hepatocellular carcinoma. Ann Surg Oncol. 2012;19:1058-65.

12. Zou G. A modified Poisson regression approach to prospective studies with binary data. Am J Epidemiol. 2004;159:702-6.

13. Greenland S. Model-based estimation of relative risks and other epidemiologic measures in studies of common outcomes and in case-control studies. Am J Epidemiol. 2004;160:301-5.

14. Barros AJ, Hirakata VN. Alternatives for logistic regression in cross-sectional studies: an empirical comparison of models that directly estimate the prevalence ratio. BMC Med Res Methodol. 2003;3:21.

15. Burt BM, Groth SS, Sada YH, Farjah F, Cornwell L, Sugarbaker DJ, et al. Utility of adjuvant chemotherapy after neoadjuvant chemoradiation and esophagectomy for esophageal cancer. Ann Surg. 2017;266:297-304.

16. Maas KW, Cuesta MA, van Berge Henegouwen MI, Roig J, Bonavina L, Rosman C, et al. Quality of life and late complications after minimally invasive compared to open esophagectomy: results of a randomized trial. World J Surg. 2015;39:1986-93.

17. Anderson JR, Cain KC, Gelber RD. Analysis of survival by tumor response. $J$ Clin Oncol. 1983;1:710-9.

18. Shapiro J, van Lanschot JJ, Hulshof $M C$, van Hagen $P$, van Berge Henegouwen MI, Wijnhoven BP, et al. Neoadjuvant chemoradiotherapy plus surgery versus surgery alone for oesophageal or junctional cancer (CROSS): long-term results of a randomised controlled trial. Lancet Oncol. 2015;16: 1090-8.

19. Oppedijk V, van der Gaast A, van Lanschot JJ, van Hagen P, van Os R, van Rij CM, et al. Patterns of recurrence after surgery alone versus preoperative chemoradiotherapy and surgery in the CROSS trials. J Clin Oncol. 2014;32: 385-91.

20. Tepper J, Krasna MJ, Niedzwiecki D, Hollis D, Reed CE, Goldberg R, et al. Phase III trial of trimodality therapy with cisplatin, fluorouracil, radiotherapy, and surgery compared with surgery alone for esophageal cancer: CALGB 9781. J Clin Oncol. 2008;26:1086-92.

21. Smyth EC, Fassan M, Cunningham D, Allum WH, Okines AF, Lampis A, et al. Effect of pathologic tumor response and nodal status on survival in the medical research council adjuvant gastric infusional chemotherapy trial. J Clin Oncol. 2016;34:2721-7.

22. Hsu PK, Chien LI, Wang LC, Chou TY. Lymphovascular invasion and extracapsular invasion are risk factors for distant recurrence after preoperative chemoradiotherapy and oesophagectomy in patients with oesophageal squamous cell carcinoma. Eur J Cardiothorac Surg. 2017;51:1188-94.

23. Saeed NA, Mellon EA, Meredith KL, Hoffe SE, Shridhar R, Frakes J, et al. Adjuvant chemotherapy and outcomes in esophageal carcinoma. J Gastrointest Oncol. 2017;8:816-24.

24. Francis AM, Sepesi B, Correa AM, Blum MA, Erasmus JJ, Lee JH, et al. The influence of histopathologic tumor viability on long-term survival and recurrence rates following neoadjuvant therapy for esophageal adenocarcinoma. Ann Surg. 2013;258:500-7.

25. Kim MP, Correa AM, Lee J, Rice DC, Roth JA, Mehran RJ, et al. Pathologic T0N1 esophageal cancer after neoadjuvant therapy and surgery: an orphan status. Ann Thorac Surg. 2010;90:884-91. 
26. Kelsen DP, Winter KA, Gunderson LL, Mortimer J, Estes NC, Haller DG, et al. Long-term results of RTOG trial 8911 (USA Intergroup 113): a random assignment trial comparison of chemotherapy followed by surgery compared with surgery alone for esophageal cancer. J Clin Oncol. 2007;25: 3719-25.

27. Lagarde SM, Phillips AW, Navidi M, Disep B, Immanuel A, Griffin SM. The presence of lymphovascular and perineural infiltration after neoadjuvant therapy and oesophagectomy identifies patients at high risk for recurrence. $\mathrm{Br} J$ Cancer. $2015 ; 113: 1427-33$

28. Schorn S, Demir IE, Reyes CM, Saricaoglu C, Samm N, Schirren R, et al. The impact of neoadjuvant therapy on the histopathological features of pancreatic ductal adenocarcinoma-a systematic review and meta-analysis. Cancer Treat Rev. 2017:55:96-106

29. Ajani JA, Mansfield PF, Janjan N, Morris J, Pisters PW, Lynch PM, et al. Multi-institutional trial of preoperative chemoradiotherapy in patients with potentially resectable gastric carcinoma. J Clin Oncol. 2004;22:2774-80.

30. Cercek A, Roxburgh CSD, Strombom P, Smith JJ, Temple LKF, Nash GM, et al. Adoption of total neoadjuvant therapy for locally advanced rectal cancer. JAMA Oncol. 2018;4:e180071

31. Goodman KA, Niedzwiecki D, Hall N, Bekaii-Saab TS, Ye X, Meyers MO. Initial results of CALGB 80803 (Alliance): a randomized phase II trial of PET scan-directed combined modality therapy for esophageal cancer. J Clin Oncol. 2017;35(4 Suppl 1).

32. Barbour AP, Rizk NP, Gonen M, Tang L, Bains MS, Rusch VW, et al. Adenocarcinoma of the gastroesophageal junction: influence of esophageal resection margin and operative approach on outcome. Ann Surg. 2007;246:1-8.

33. Schipper PH, Cassivi SD, Deschamps C, Rice DC, Nichols FC III, Allen MS, et al. Locally recurrent esophageal carcinoma: when is re-resection indicated? Ann Thorac Surg. 2005;80:1001-6.

34. Rueth NM, Parsons HM, Habermann EB, Groth SS, Virnig BA, Tuttle TM, et al. The long-term impact of surgical complications after resection of stage I nonsmall cell lung cancer: a population-based survival analysis. Ann Surg. 2011;254:368-74.

35. Bilimoria KY, Bentrem DJ, Stewart AK, Winchester DP, Ko CY. Comparison of commission on cancer-approved and -nonapproved hospitals in the United States: implications for studies that use the national cancer data base. J Clin Oncol. 2009;27:4177-81.

36. Lerro CC, Robbins AS, Phillips JL, Stewart AK. Comparison of cases captured in the national cancer data base with those in population-based central cancer registries. Ann Surg Oncol. 2013;20:1759-65.

Key Words: esophageal neoplasms, esophagectomy, neoadjuvant therapy, pathology

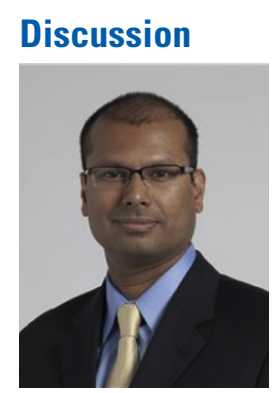

Dr Siva Raja (Cleveland, Ohio). I thank the Association for the invitation to discuss the work.

I have no relevant disclosures. The facts that esophageal adenocarcinoma is an aggressive disease and the treatment is morbid are not in question. What is in question is what population we currently do not help and how can we do a better job. In this study, the authors use the NCDB data set to examine the impact of response to neoadjuvant therapy on long-term survival. They show a graded improvement of survival with a graded increase in response to therapy. They also show that nodal status trumps response in primary therapy. However, with big data we could have big holes in the data, and this is primarily due to a lack of granularity and the retrospective nature of the data set.

We and others have shown that in the setting of primary response to treatment, characterizing percent of viable tumor cells as a continuous variable can allow you to separate the signal from the noise. However, those were individual studies with small patient populations relative to the larger study that you are presenting today.

Because it is easier to critique than do research, I am going to start by congratulating you on a study well done and I have 2 questions for you, Dr Groth. First, information about response to your study only provides answers after being subjected to an esophagectomy. Did you have any sense from the study if there were any predictive factors such as tumor grade, inability to complete treatment, et cetera, that predicted a poor response? It would be nice to know who was not going to respond and think about their algorithm differently from the beginning as opposed to try to change course in the middle like in the anecdote you suggested.

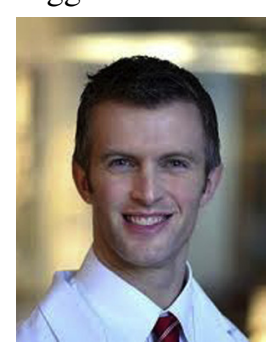

Dr Shawn S. Groth (Houston, Tex). Thank you, Dr Raja. One of the limitations of this study is an inherent limitation of $\mathrm{NCDB}$ - we are only provided with pretreatment clinical staging. We don't have any information on post-treatment clinical staging, which, as you mentioned, would be beneficial in terms of making treatment decisions prior to committing a patient to esophagectomy. Despite this limitation, the accuracy of post-treatment clinical staging is imperfect. By using pathologic staging, we have $100 \%$ accuracy regarding post-treatment primary tumor and nodal response. To answer your question, the focus of our study was on the prognostic implications of primary tumor and nodal response to neoadjuvant therapy. In our multivariable models, we adjusted for covariates such as tumor grade, but we did not specifically isolate covariates that predict poor response to therapy.

Dr Raja. The second question is, nodal stage trumps tumor stage. Have you looked at a more refined population such as those with ypn0 to see if there is any response to therapy between chemotherapy only and chemotherapy and radiotherapy to see if in that population the effect on primary tumor has some benefit in long-term survival?

Dr Groth. We didn't look at the effect of tumor and nodal response on survival among patients treated with chemotherapy alone because they comprised only a small percentage of esophageal cancer patients in the NCDB. In terms of response to treatment, yes; there was an association between use of radiation and improved primary tumor response in node-negative patients. 


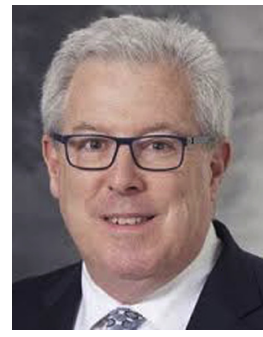

Dr Malcolm M. DeCamp (Madison, Wis). Congratulations on an important study. I wanted to get your thoughts about this issue of pathologic response, because that's a snapshot in time and it depends on the timing from completion of therapy to the day you go to the operating room, and it's obviously a biologic continuum. So does the NCDB have data on the interval from when they completed therapy to surgery? There are articles from colleagues across town in Houston that suggest perhaps the adjuvant window can be extended longer from completion of therapy if the goal is to see that true pathologic complete response. Is there a way to assess for those near-complete responders?

Dr Groth. The time interval between the completion of radiation therapy and the date of surgery can be calculated by using NCDB data. However, we did not specifically look at the time interval between completion of chemoradiation therapy and esophagectomy and whether or not longer intervals increased the percentage of complete responders as compared to partial responders.

Unidentified Speaker. You showed 3 curves, right? You have the complete pathologic responders, you have the intermediate, we will say, and then you have the nonresponders, but really there should be 9 curves. What I mean by that is, where you start actually has significant impact on the OS. So if the patient has T3N0 and receives chemoradiation therapy and is a complete responder, the likelihood for cure is a lot better than somebody with $\mathrm{TN} 2$ or N3.

So I think what happens is the NCDB database doesn't allow you to do that, but others have shown that if you take T3N0s and give them chemoradiation and they are complete responders, most of those patients will have $50 \%$ cure rates or better, whereas in the old staging system it was sort of N1 or N1A, meaning celiac or supraclavicular nodes. If you took a person who had N1 disease, either celiac access or supraclavicular nodes, even if they were "complete pathological responders," their overall failure rate was higher than people who had a T3N0 start.

So I think one of the problems with this database is you won't really know accurately where people started. You only know where they kind of finished when you look at the final pathology, right?

Dr Groth. All that we know from the NCBD is the pretreatment clinical stage and the final pathologic stage. That's all we get. Of course, clinical staging is not $100 \%$ accurate.

Unidentified Speaker. But the preoperative staging can vary from institution to institution, in other words, how hard you look.

Dr Groth. Yes, of course. The NCDB isn't granular enough to provide information on what was used, such as
EUS, PET, or staging laparoscopy, to establish the clinical stage. There's no information on the quality of pretreatment staging. It is what it is. That's the nature of the data. Though it may be perceived as a limitation, this is reflective of everyday practice around the country, which makes our results generalizable.

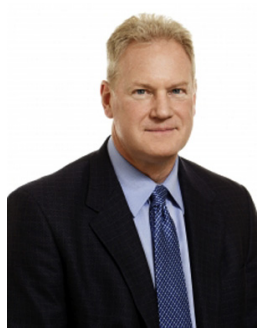

Dr Steven R. DeMeester (Portland, Ore). Just a plea for caution. The data are interesting, but for the last decade we have been on this quest of pathologic complete response, centers like MD Anderson cranking up the radiation and trying everything they can to achieve complete pathologic response. But in reality, most of these patients are dying of systemic disease, and complete pathologic response is kind of a fog, because it depends on how hard you look for it. If you do immunohistochemistry versus just cutting a node and using hematoxylin-eosin or if you go way back to Jerry Sullivan's work and you do bone marrow aspirations on people who supposedly have pathologic complete response, you find viable tumor cells in the bone marrow. So we are chasing after the wind with this pathologic complete response when what we really need is systemic control. I think we have to be careful, because chemotherapy regimens are never going to provide the pathologic complete response rates that we are getting with the addition of radiation treatment, yet radiation doesn't help the systemic disease problem that we are facing.

So I think we have to shift our thinking a bit going forward, and I would like your assessment of systemic disease. Do you have any information on systemic disease in these 2 groups that received chemo only versus chemoradiation?

Dr Groth. To respond to your first point, I wasn't saying that we should target a complete pathologic response before esophagectomy, but we should do the best that we can within reason to try to sterilize their lymph nodes. By no means am I trying to imply that patients who have suspected residual nodal disease be treated with endless cycles of chemotherapy until they sterilize their nodes. As you mentioned, most patients die of systemic disease and better systemic control is needed. However, if we've learned anything from history, the majority of patients who have adjuvant therapy as part of their pretreatment care plan can't complete it. If systemic therapy is part of the treatment plan, we should consider optimizing its use prior to esophagectomy.

Unfortunately, the NCDB does collect information on recurrences, but it doesn't provide those that data to researchers because there's a lot of missing data. Consequently, we can't look at systemic failures in patients 
treated with chemoradiation therapy versus chemotherapy alone.

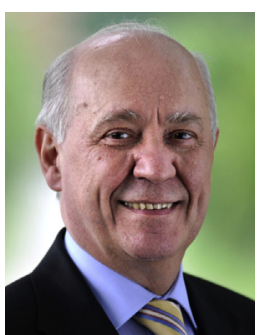

Dr Toni Lerut (Leuven, Belgium). Excellent presentation, but I think the picture is only complete if you know also, and I don't know whether the database allows you to know that, the dropouts and whether there is a difference between dropouts in the chemo versus chemoradiation. We have the impression that there are more dropouts in the chemo rads than in the chemo.

Dr Groth. We don't have information on dropouts per se with the NCDB. We do have information on missing data, and perhaps some of the patients with missing data didn't complete all of their assigned therapy. We accounted for missing data with our statistical methods, although we did not directly compare the amount of missing data in the chemotherapy alone versus chemoradiation groups.

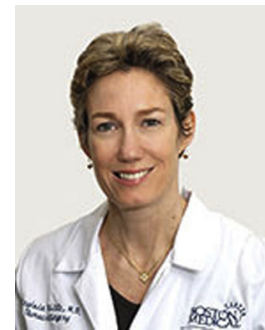

Dr Virginia Litle (Boston, Mass). I just want to make a little shameless plug for some of our own research that we presented at the Society of Thoracic Surgeons on using circulating cell-free tumor DNA to look at patients who are responding to therapy or for early recurrence. We should be focusing more effort on our biomarkers and identifying the subsets to help in this decision-making treating algorithm. Otherwise we may be just overtreating patients even with unnecessary resection.

Readers who found these articles interesting may also like to read the following papers found in recent and future issues of our sister publications, Seminars in Thoracic and Cardiovascular Surgery and Operative Techniques in Thoracic and Cardiovascular Surgery!

Thoracic: Esophageal Cancer

ORIGINAL SUBMISSION: Delay in Oral Feeding is Associated With a Decrease in Anastomotic Leak Following Transhiatal Esophagectomy. James Speicher. Semin Thoracic Surg 2018: Volume 30, Issue 4.

Editorial Commentary: To eat or not to eat: Does delaying oral feeding decrease anastomotic leaks? Jules Lin. Semin Thoracic Surg 2018: Volume 30, Issue 4.

ORIGINAL SUBMISSION: Esophagectomy in Patients with Human Immunodeficiency Virus and Acquired Immune Deficiency Syndrome: A Viable Option. Michael Mwachiro. Semin Thoracic Surg 2018:116-121. 
TABLE E1. Complete model results from Cox regression for the overall cohort

\begin{tabular}{|c|c|}
\hline & Hazard ratio $(95 \%$ CI $)$ \\
\hline \multicolumn{2}{|l|}{ Treatment response } \\
\hline No response & Ref \\
\hline Partial response & $0.80(0.71-0.91)$ \\
\hline Complete response & $0.54(0.46-0.63)$ \\
\hline \multicolumn{2}{|l|}{ Age, y } \\
\hline$\leq 55$ & Ref \\
\hline $56-60$ & $1.04(0.92-1.19)$ \\
\hline $61-65$ & $1.14(0.98-1.32)$ \\
\hline$>65$ & $1.19(1.01-1.39)$ \\
\hline Female sex & $0.86(0.73-1.01)$ \\
\hline Nonwhite race & $1.29(0.92-1.80)$ \\
\hline \multicolumn{2}{|l|}{ Insurance } \\
\hline Insured & Ref \\
\hline Medicare/Medicaid & $1.26(1.10-1.45)$ \\
\hline Other & $1.39(1.04-1.87)$ \\
\hline Income $>\$ 63,000 *$ & $0.93(0.80-1.09)$ \\
\hline Education* & $0.83(0.73-0.96)$ \\
\hline \multicolumn{2}{|l|}{ Rurality } \\
\hline Metropolitan & Ref \\
\hline Urban & $1.01(0.88-1.17)$ \\
\hline Rural & $1.00(0.86-1.18)$ \\
\hline \multicolumn{2}{|l|}{ Charlson index } \\
\hline 0 & Ref \\
\hline 1 & $1.17(1.05-1.32)$ \\
\hline$\geq 2$ & $1.14(0.90-1.44)$ \\
\hline \multicolumn{2}{|l|}{ Facility } \\
\hline Academic/research & Ref \\
\hline Comprehensive cancer center & $1.16(1.02-1.32)$ \\
\hline Other & $0.98(0.83-1.16)$ \\
\hline Grade, poor/undifferentiated & $1.29(1.14-1.45)$ \\
\hline Adjuvant therapy & $0.80(0.67-0.97)$ \\
\hline
\end{tabular}

